PROCEEDINGS OF THE

AMERICAN MATHEMATICAL SOCIETY

Volume 132, Number 11, Pages 3195-3201

S 0002-9939(04)07610-5

Article electronically published on June 16, 2004

\title{
TWO ESTIMATES FOR CURVES IN THE PLANE
}

\author{
DANIEL M. OBERLIN
}

(Communicated by Andreas Seeger)

\begin{abstract}
We obtain a Fourier transform estimate and an $L^{3 / 2}\left(\mathbb{R}^{2}\right)-L^{3}\left(\mathbb{R}^{2}\right)$ convolution estimate for certain measures on a class of convex curves in the plane.
\end{abstract}

\section{§1. INTRODUCTION}

This is the third in a series of papers concerned with estimates for operators associated with measures on a certain class of curves in the plane. The curves are just graphs $\Gamma=\{(x, \phi(x)): a \leq x<b\}$ where $\phi^{(j)}(a)=0$ for $j=0,1,2$ and $\phi^{\prime \prime}>0, \phi^{(3)} \geq 0$ on $(a, b)$. Our previous results deal with the affine arclength measure $\phi^{\prime \prime}(x)^{1 / 3} d x$ on $\Gamma$. They are

Theorem 1 ([7]). Writing $\lambda$ for affine arclength on $\Gamma$, there is the estimate

$$
\left\|\lambda * \chi_{E}\right\|_{L^{3}\left(\mathbb{R}^{2}\right)} \leq 12^{1 / 3}\left\|\chi_{E}\right\|_{3 / 2}
$$

for any measurable $E \subseteq \mathbb{R}^{2}$.

Theorem $2([8])$. If $1 \leq p<\frac{4}{3}$ and $\frac{1}{p}+\frac{1}{3 q}=1$, there is a constant $C=C(p)$ such that the estimate

$$
\left(\int_{a}^{b}|\widehat{f}(t, \phi(t))|^{q} \phi^{\prime \prime}(t)^{\frac{1}{3}} d t\right)^{\frac{1}{q}} \leq C(p)\|f\|_{L^{p}\left(\mathbb{R}^{2}\right)}
$$

holds.

(Shortly after [8] appeared, it was pointed out to the author that Theorem 2 is a consequence of Theorem 2 in Sjölin's paper [9.) Part of the novelty of Theorems 1 and 2 is that the estimates they provide are uniform over the class of curves under consideration. In particular, the constant $C(p)$ in Theorem 2 is independent of $\phi$. Convolution and Fourier restriction estimates like those in Theorems 1 and 2 are well known when the associated curves have nonvanishing curvature. Drury ([3]) pointed out that the damping factor $\phi^{\prime \prime}(x)^{1 / 3}$ could compensate for flatness in such estimates. But his and subsequent results (see, e.g., 1]) gave bounds depending on certain ancillary constants. The (quite simple) proofs of Theorems 1 and 2

Received by the editors March 27, 2002.

2000 Mathematics Subject Classification. Primary 42B20.

Key words and phrases. Fourier transform, convolution.

The author was partially supported by a grant from the National Science Foundation.

(C)2004 American Mathematical Society 
have no such dependence. The shortcoming of Theorem 1 is that it holds only for characteristic functions $\chi_{E}$ : a more natural estimate would be

$$
\|\lambda * f\|_{L^{3}\left(\mathbb{R}^{2}\right)} \leq C\|f\|_{L^{3 / 2}\left(\mathbb{R}^{2}\right)}
$$

for nonnegative and measurable functions $f$ on $\mathbb{R}^{2}$ and for some absolute constant $C$. We have been unable to prove or disprove such an estimate. Our next result is a weaker substitute.

Theorem 3. With $\phi$ as above, write $\omega(x)$ for the function $\frac{\phi^{\prime}(x)^{2}}{\phi(x)}$ and $\nu$ for the measure on $\Gamma$ given by $d \nu=\omega(x)^{1 / 3} d x$. Then there is an absolute constant $C$ such that the estimate

$$
\|\nu * f\|_{L^{3}\left(\mathbb{R}^{2}\right)} \leq C\|f\|_{L^{3 / 2}\left(\mathbb{R}^{2}\right)}
$$

holds for nonnegative measurable functions $f$ on $\mathbb{R}^{2}$.

(One can check that Theorem 3 is weaker than (1) by verifying, as we do in the proof of Theorem 4 , that the inequality $\omega(x) \leq 2 \phi^{\prime \prime}(x)$ follows from the hypotheses on $\phi$.)

There is also a related Fourier transform estimate.

Theorem 4. There is an absolute constant $C$ such that the following holds: with $\phi$ and $\omega$ as above, with $[c, d] \subseteq[a, b)$, and with $\zeta, \eta \in \mathbb{R}$, we have the inequality

$$
\left|\int_{c}^{d} e^{i(\zeta x+\eta \phi(x))} \omega(x)^{1 / 2} d x\right| \leq \frac{C}{|\eta|^{1 / 2}} .
$$

If $\zeta=0$, then the change of variable $t=\phi(x)^{1 / 2}$ shows the conclusion of Theorem 4 to follow trivially from Van der Corput's lemma applied to $\int e^{i \eta t^{2}} d t$. On the other hand, a stronger estimate

$$
\left|\int_{c}^{d} e^{i(\zeta x+\eta \phi(x))} \omega(x)^{1 / 2+i s} d x\right| \leq \frac{C(s)}{|\eta|^{1 / 2}},
$$

with $C(s)$ growing, say, polynomially in $|s|$ (which we have been unable to obtain) would yield a proof of Theorem 3 different from the one we present. Results like Theorem 3, but for nondegenerate curves and without a uniform constant, date back at least to [5]. Fourier transform estimates such as that of Theorem 4, but for nondegenerate curves, are easy consequences of Van der Corput's lemma. The remainder of this note is organized as follows: $\S 2$ contains the proof of Theorem 3 , and $\S 3$ contains the proof of Theorem 4.

\section{$\S 2$. Proof of Theorem 3}

Theorem 3 is proved by adapting the method of Drury and Guo in [4]. The proof requires an elementary lemma.

Lemma 1. With $\phi$ and $[a, b)$ as above, suppose $h>0$ and $x, x-h \in[a, b)$. Then

$$
\frac{\phi^{\prime}(x) \phi^{\prime}(x-h)}{\phi(x)^{1 / 2} \phi(x-h)^{1 / 2}\left(\phi^{\prime}(x)-\phi^{\prime}(x-h)\right)} \leq \frac{5}{|h|} .
$$


Proof of Lemma 1. Since $\phi(a)=\phi^{\prime}(a)=0$ it follows, for example, that $\phi(x)=$ $\int_{a}^{x}(x-t) \phi^{\prime \prime}(t) d t$. Thus the conclusion of Lemma 1 is equivalent to the inequality

$$
\begin{aligned}
& h \int_{a}^{x} \phi^{\prime \prime}(t) d t \int_{a}^{x-h} \phi^{\prime \prime}(t) d t \\
& \quad \leq 5\left(\int_{a}^{x}(x-t) \phi^{\prime \prime}(t) d t\right)^{1 / 2}\left(\int_{a}^{x-h}(x-h-t) \phi^{\prime \prime}(t) d t\right)^{1 / 2} \int_{x-h}^{x} \phi^{\prime \prime}(t) d t .
\end{aligned}
$$

It is therefore enough to establish the two inequalities

$$
\begin{aligned}
& h\left(\int_{a}^{x-h} \phi^{\prime \prime}(t) d t\right)^{2} \\
& \quad \leq 2\left(\int_{a}^{x}(x-t) \phi^{\prime \prime}(t) d t\right)^{1 / 2}\left(\int_{a}^{x-h}(x-h-t) \phi^{\prime \prime}(t) d t\right)^{1 / 2} \int_{x-h}^{x} \phi^{\prime \prime}(t) d t
\end{aligned}
$$

and

$$
\begin{aligned}
& h \int_{x-h}^{x} \phi^{\prime \prime}(t) d t \int_{a}^{x-h} \phi^{\prime \prime}(t) d t \\
& \quad \leq 3\left(\int_{a}^{x}(x-t) \phi^{\prime \prime}(t) d t\right)^{1 / 2}\left(\int_{a}^{x-h}(x-h-t) \phi^{\prime \prime}(t) d t\right)^{1 / 2} \int_{x-h}^{x} \phi^{\prime \prime}(t) d t .
\end{aligned}
$$

With no loss of generality, assume $\phi^{\prime \prime}(x-h)=1$. Since $\phi^{\prime \prime}$ is increasing, it follows that $h \leq \int_{x-h}^{x} \phi^{\prime \prime}(t) d t$. Since

$$
\int_{a}^{x-h}(x-h-t) \phi^{\prime \prime}(t) d t \leq \int_{a}^{x}(x-t) \phi^{\prime \prime}(t) d t
$$

inequality (2) will follow from

$$
\left(\int_{a}^{x-h} \phi^{\prime \prime}(t) d t\right)^{2} \leq 2 \int_{a}^{x-h}(x-h-t) \phi^{\prime \prime}(t) d t .
$$

To see this, let $\epsilon=\int_{a}^{x-h} \phi^{\prime \prime}(t) d t$. Since $\phi^{\prime \prime}(t) \leq \phi^{\prime \prime}(x-h)=1$ if $a \leq t \leq x-h$, the RHS of (4) is minimized when $\phi^{\prime \prime}(t)=\chi_{[x-h-\epsilon, x-h]}$ on $[a, x-h]$. This minimum is $\epsilon^{2} / 2$, and so (4) holds. Now, if $a \leq x-2 h,(3)$ will follow from the inequalities

$$
h \int_{a}^{x-2 h} \phi^{\prime \prime}(t) d t \leq\left(\int_{a}^{x}(x-t) \phi^{\prime \prime}(t) d t\right)^{1 / 2}\left(\int_{a}^{x-h}(x-h-t) \phi^{\prime \prime}(t) d t\right)^{1 / 2}
$$

and

$$
h \int_{x-2 h}^{x-h} \phi^{\prime \prime}(t) d t \leq 2\left(\int_{a}^{x}(x-t) \phi^{\prime \prime}(t) d t\right)^{1 / 2}\left(\int_{a}^{x-h}(x-h-t) \phi^{\prime \prime}(t) d t\right)^{1 / 2} .
$$

The first of these is clear since both of $x-t$ and $x-t-h$ exceed $h$ on $[a, x-2 h]$. For the second, note that

$$
h^{2} \leq 2 \int_{x-h}^{x}(x-t) \phi^{\prime \prime}(t) d t \leq 2 \int_{a}^{x}(x-t) \phi^{\prime \prime}(t) d t
$$

since $\phi^{\prime \prime}$ is nondecreasing and $\phi^{\prime \prime}(x-h)=1$. Thus the desired inequality follows from (4). A slight modification handles the case $x-2 h<a$ and completes the proof of the lemma. 
As previously mentioned, the proof of Theorem 3 is an adaptation of the method of [4. For $f \geq 0$ we need to estimate

$$
\|\nu * f\|_{L^{3}\left(\mathbb{R}^{2}\right)}^{3}=\int \cdots \int \prod_{j=1}^{3}\left(f\left(x-t_{j}, y-\phi\left(t_{j}\right)\right) \omega^{1 / 3}\left(t_{j}\right)\right) d t_{1} d t_{2} d t_{3} d x d y
$$

where the $t_{j}$ integrals are over $[a, b)$ and the $x$ and $y$ integrals are over $\mathbb{R}$. The change of variables $x_{j}=x-t_{j}$ leads to

$$
\int \cdots \int \prod_{j=1}^{3}\left(f\left(x_{j}, y-\phi\left(x-x_{j}\right)\right) \omega^{1 / 3}\left(x-x_{j}\right)\right) d x d y d x_{1} d x_{2} d x_{3}
$$

where the $x_{j}$ integrals and the $y$ integral are over $\mathbb{R}$ and the $x$ integral is over $\bigcap_{j}\left(x_{j}+[a, b)\right)$. The idea of [4] is to fix temporarily the $x_{j}$ and obtain an estimate of the $x y$ integral. Accordingly, we let $g_{j}(z)=f\left(x_{j}, z\right)$ and consider

$$
S\left(g_{1}, g_{2}, g_{3}\right)=\iint \prod_{j=1}^{3}\left(g_{j}\left(y-\phi\left(x-x_{j}\right)\right) \omega^{1 / 3}\left(x-x_{j}\right)\right) d x d y .
$$

The desired estimate is

$$
S\left(g_{1}, g_{2}, g_{3}\right) \leq \frac{C\left\|g_{1}\right\|_{L^{3 / 2}(\mathbb{R})}\left\|g_{2}\right\|_{L^{3 / 2}(\mathbb{R})}\left\|g_{3}\right\|_{L^{3 / 2}(\mathbb{R})}}{\left|\left(x_{2}-x_{3}\right)\left(x_{3}-x_{1}\right)\left(x_{1}-x_{2}\right)\right|^{1 / 3}} .
$$

Combining (6) with the following estimate of Christ ([2], Proposition 2.2),

$$
\begin{aligned}
\iiint \frac{\left|h_{1}\left(x_{1}\right) h_{2}\left(x_{2}\right) h_{3}\left(x_{3}\right)\right|}{\left|\left(x_{2}-x_{3}\right)\left(x_{3}-x_{1}\right)\left(x_{1}-x_{2}\right)\right|^{1 / 3}} d x_{1} d x_{2} d x_{3} \\
\leq C\left\|h_{1}\right\|_{L^{3 / 2}(\mathbb{R})}\left\|h_{2}\right\|_{L^{3 / 2}(\mathbb{R})}\left\|h_{3}\right\|_{L^{3 / 2}(\mathbb{R})},
\end{aligned}
$$

shows that (5) is bounded by $C\|f\|_{L^{3 / 2}\left(\mathbb{R}^{2}\right)}^{3}$. Inequality (6) follows from an interpolation based on the three estimates $(7.1),(7.2)$, and (7.3), where (7.1) is

$$
\begin{gathered}
\iint\left(g_{1}\left(y-\phi\left(x-x_{1}\right)\right) \prod_{j=2}^{3}\left(g_{j}\left(y-\phi\left(x-x_{j}\right)\right) \omega^{1 / 2}\left(x-x_{j}\right)\right) d x d y\right. \\
\leq \frac{C\left\|g_{1}\right\|_{L^{\infty}(\mathbb{R})}\left\|g_{2}\right\|_{L^{1}(\mathbb{R})}\left\|g_{3}\right\|_{L^{1}(\mathbb{R})}}{\left|x_{2}-x_{3}\right|}
\end{gathered}
$$

and (7.2) and (7.3) are analogous. To see (7.1) note that

$$
\begin{aligned}
\iint & \prod_{j=2}^{3}\left(g_{j}\left(y-\phi\left(x-x_{j}\right)\right) \omega^{1 / 2}\left(x-x_{j}\right)\right) d x d y \\
= & \iint g_{2}\left(y-\phi\left(x-x_{2}\right)\right) g_{3}\left(y-\phi\left(x-x_{3}\right)\right) \\
& \cdot \frac{\phi^{\prime}\left(x-x_{2}\right) \phi^{\prime}\left(x-x_{3}\right)}{\phi\left(x-x_{2}\right)^{1 / 2} \phi\left(x-x_{3}\right)^{1 / 2}\left|\phi^{\prime}\left(x-x_{2}\right)-\phi^{\prime}\left(x-x_{3}\right)\right|} \\
& \cdot\left|\phi^{\prime}\left(x-x_{2}\right)-\phi^{\prime}\left(x-x_{3}\right)\right| d x d y \\
\leq & \frac{5\left\|g_{2}\right\|_{L^{1}(\mathbb{R})}\left\|g_{3}\right\|_{L^{1}(\mathbb{R})}}{\left|x_{2}-x_{3}\right|}
\end{aligned}
$$


by Lemma 1 and the fact that the Jacobian determinant for the one-to-one mapping $(x, y) \mapsto\left(y-\phi\left(x-x_{2}\right), y-\phi\left(x-x_{3}\right)\right)$ has absolute value $\left|\phi^{\prime}\left(x-x_{2}\right)-\phi^{\prime}\left(x-x_{3}\right)\right|$.

\section{§3. Proof of Theorem 4}

We begin with a pair of lemmas.

Lemma 2. Suppose $\psi$ is a real-valued continuously differentiable function on a closed interval $I$ such that $\psi$ and $\psi^{\prime}$ are of constant sign on I. Then

$$
\left|\int_{I} e^{i r u} \psi(u) d u\right| \leq 5 \sup \left\{\left|\int_{J} \psi\right|: J \text { is a subinterval of } I \text { with length } \leq \frac{1}{|r|}\right\} \text {. }
$$

This is Lemma 1 in [6]. The change of variable $u=\alpha(x)$ yields the next lemma.

Lemma 3. With $\psi$ as in Lemma 2, suppose that $\alpha$ is a twice continuously differentiable function on I such that $\alpha^{\prime}$ and $\alpha^{\prime} \psi^{\prime}-\psi \alpha^{\prime \prime}$ are of constant sign on I. Then

$$
\left|\int_{I} e^{i \alpha(x)} \psi(x) d x\right| \leq 5 \sup \left\{\left|\int_{x_{0}}^{x_{1}} \psi\right|:\left|\alpha\left(x_{1}\right)-\alpha\left(x_{0}\right)\right| \leq 1\right\} .
$$

Theorem 4 is the estimate

$$
\left|\int_{c}^{d} e^{i(\zeta x+\eta \phi(x))} \omega(x)^{1 / 2} d x\right| \leq \frac{C}{|\eta|^{1 / 2}} .
$$

If $\zeta$ and $\eta$ have the same sign, then one can apply an easy argument based on the change of variable $t=\phi(x)^{1 / 2}$. So we will assume that $\zeta$ and $\eta$ have opposite signs. Furthermore, it is sufficient to establish (8) under the additional hypothesis that if $\alpha(x)=\zeta x+\eta \phi(x)$, then $\alpha^{\prime}$ is of constant sign on $[c, d]$. Of course we intend to apply Lemma 3 with $\psi=\omega^{1 / 2}=\frac{\phi^{\prime}}{\phi^{1 / 2}}$, and so we need to check that $\alpha^{\prime} \psi^{\prime}-\psi \alpha^{\prime \prime}$ is of constant sign. Now

$$
\alpha^{\prime} \psi^{\prime}-\psi \alpha^{\prime \prime}=\left(\zeta+\eta \phi^{\prime}\right) \frac{\phi \phi^{\prime \prime}-\frac{1}{2}\left(\phi^{\prime}\right)^{2}}{\phi^{3 / 2}}-\frac{\phi^{\prime}}{\phi^{1 / 2}}\left(\eta \phi^{\prime \prime}\right)=-\frac{\eta}{2} \frac{\left(\phi^{\prime}\right)^{3}}{\phi^{3 / 2}}+\zeta \frac{\phi \phi^{\prime \prime}-\frac{1}{2}\left(\phi^{\prime}\right)^{2}}{\phi^{3 / 2}} .
$$

Since

$$
\begin{gathered}
\phi^{\prime}(x)=\int_{a}^{x}(x-t) \phi^{(3)}(t) d t \\
\leq\left(\int_{a}^{x}(x-t)^{2} \phi^{(3)}(t) d t\right)^{\frac{1}{2}}\left(\int_{a}^{x} \phi^{(3)}(t) d t\right)^{\frac{1}{2}}=\sqrt{2} \phi(x)^{1 / 2} \phi^{\prime \prime}(x)^{1 / 2},
\end{gathered}
$$

the fact that $\zeta$ and $\eta$ have opposite signs shows that $\alpha^{\prime} \psi^{\prime}-\psi \alpha^{\prime \prime}$ has constant sign. To apply Lemma 2 we assume that $\left[x_{0}, x_{1}\right] \subseteq[c, d]$ and that $\mid \eta\left(\phi\left(x_{1}\right)-\phi\left(x_{0}\right)\right)+$ $\zeta\left(x_{1}-x_{0}\right) \mid \doteq \epsilon \leq 1$, and we will then establish the inequality

$$
\int_{x_{0}}^{x_{1}} \frac{\phi^{\prime}(x)}{\phi(x)^{1 / 2}} d x \leq \frac{2}{|\eta|^{1 / 2}} .
$$

Assume without loss of generality that $\eta>0$, and assume for the moment that

$$
\phi^{\prime} \geq-\frac{\zeta}{\eta}
$$

on $\left[x_{0}, x_{1}\right]$. (This inequality or its opposite must hold on $\left[x_{0}, x_{1}\right]$ because the sign of $\alpha^{\prime}$ is constant on $[c, d]$.) Then

$$
\eta\left(\phi\left(x_{1}\right)-\phi\left(x_{0}\right)\right)=-\zeta\left(x_{1}-x_{0}\right)+\epsilon
$$


and so

$$
-\frac{\zeta}{\eta}=\frac{\eta\left(\phi\left(x_{1}\right)-\phi\left(x_{0}\right)\right)-\epsilon}{\eta\left(x_{1}-x_{0}\right)} .
$$

Thus (since $\phi^{\prime}$ is increasing) (10) holds on $\left[x_{0}, x_{1}\right]$ if and only if

$$
\phi^{\prime}\left(x_{0}\right) \geq \frac{\phi\left(x_{1}\right)-\phi\left(x_{0}\right)}{x_{1}-x_{0}}-\frac{\epsilon}{\eta\left(x_{1}-x_{0}\right)},
$$

which is equivalent to

$$
\frac{\epsilon}{\eta} \geq \phi\left(x_{1}\right)-\phi\left(x_{0}\right)-\phi^{\prime}\left(x_{0}\right)\left(x_{1}-x_{0}\right)=\int_{x_{0}}^{x_{1}}\left(x_{1}-t\right) \phi^{\prime \prime}(t) d t .
$$

If, instead of (10), we assume

$$
\phi^{\prime} \leq-\frac{\zeta}{\eta}
$$

on $\left[x_{0}, x_{1}\right]$, then it follows similarly that

$$
\frac{\epsilon}{\eta} \geq \phi\left(x_{1}\right)-\phi\left(x_{0}\right)-\phi^{\prime}\left(x_{0}\right)\left(x_{1}-x_{0}\right)=\int_{x_{0}}^{x_{1}}\left(t-x_{0}\right) \phi^{\prime \prime}(t) d t .
$$

Since $\phi^{\prime \prime}$ is nondecreasing, $\int_{x_{0}}^{x_{1}}\left(x_{1}-t\right) \phi^{\prime \prime}(t) d t \leq \int_{x_{0}}^{x_{1}}\left(t-x_{0}\right) \phi^{\prime \prime}(t) d t$, and therefore

$$
\left(\int_{x_{0}}^{x_{1}}\left(x_{1}-t\right) \phi^{\prime \prime}(t) d t\right)^{1 / 2} \leq \frac{\epsilon^{1 / 2}}{|\eta|^{1 / 2}} \leq \frac{1}{|\eta|^{1 / 2}}
$$

Thus (9) will follow from

$$
\int_{x_{0}}^{x_{1}} \frac{\phi^{\prime}(x)}{\phi(x)^{1 / 2}} d x \leq 2\left(\int_{x_{0}}^{x_{1}}\left(x_{1}-t\right) \phi^{\prime \prime}(t) d t\right)^{1 / 2} .
$$

For $x \in(a, b)$ consider

$$
\inf \left\{\frac{\phi^{\prime}(x)-\phi^{\prime}\left(x_{0}\right)}{\left(\int_{x_{0}}^{x}(x-t) \phi^{\prime \prime}(t) d t\right)^{1 / 2}}: a \leq x_{0}<x\right\} .
$$

A computation shows that

$$
\frac{d}{d x_{0}}\left(\frac{\left(\phi^{\prime}(x)-\phi^{\prime}\left(x_{0}\right)\right)^{2}}{\int_{x_{0}}^{x}(x-t) \phi^{\prime \prime}(t) d t}\right)
$$

has the same sign as $\int_{x_{0}}^{x} \phi^{\prime \prime}(t)\left(\left(x-x_{0}\right)-2(x-t)\right) d t$, which is nonnegative since $\phi^{\prime \prime}$ is positive and nondecreasing. Thus the infimum above is realized when $x_{0}=a$, and therefore

$$
\inf \left\{\frac{\phi^{\prime}(x)-\phi^{\prime}\left(x_{0}\right)}{\left(\int_{x_{0}}^{x}(x-t) \phi^{\prime \prime}(t) d t\right)^{1 / 2}}: a \leq x_{0}<x\right\}=\frac{\phi^{\prime}(x)}{\phi(x)^{1 / 2}} .
$$

On the other hand, for fixed $x_{0}$,

$$
\frac{d}{d x}\left(\int_{x_{0}}^{x}(x-t) \phi^{\prime \prime}(t) d t\right)^{1 / 2}=\frac{\phi^{\prime}(x)-\phi^{\prime}\left(x_{0}\right)}{2\left(\int_{x_{0}}^{x}(x-t) \phi^{\prime \prime}(t) d t\right)^{1 / 2}} .
$$


Thus, by (12),

$$
\int_{x_{0}}^{x_{1}} \frac{\phi^{\prime}(x)}{\phi(x)^{1 / 2}} d x \leq 2 \int_{x_{0}}^{x_{1}} \frac{d}{d x}\left(\int_{x_{0}}^{x}(x-t) \phi^{\prime \prime}(t) d t\right)^{1 / 2} d x=2\left(\int_{x_{0}}^{x_{1}}(x-t) \phi^{\prime \prime}(t) d t\right)^{1 / 2} \text {. }
$$

This is (11), and so the proof of Theorem 4 is complete.

\section{REFERENCES}

1. Y. Choi, Convolution operators with affine arclength measures on plane curves, J. Korean Math. Soc. 36 (1999), 193-207. MR 2000a:42021

2. M. Christ, On the restriction of the Fourier transform to curves: endpoint results and the degenerate case, Trans. Amer. Math. Soc. 287 (1985), 223-238. MR 87b:42018

3. S.W. Drury, Degenerate curves and harmonic analysis, Math. Proc. Camb. Phil. Soc. 108 (1990), 89-96. MR 91h:42021

4. S. W. Drury and K. Guo, Convolution estimates related to surfaces of half the ambient dimension, Math. Proc. Camb. Phil. Soc. 110 (1991), 151-159. MR 92j:42012

5. W. Littman, $L^{p}-L^{q}$ estimates for singular integral operators, Proc. Sympos. Pure Math. 23 (1971), 479-481. MR 50:10909

6. D.M. Oberlin, Oscillatory integrals with polynomial phase, Math. Scand. 69 (1991), 45-56. MR 93c:41048

7. _ Convolution with affine arclength measures in the plane, Proc. Amer. Math. Soc. 127 (1999), 3591-3592. MR 2000c:42016

8. Soc. 129 (2001), 3303-3305. MR 2002g:42013

9. P. Sjölin, Fourier multipliers and estimates of the Fourier transform carried by smooth curves in $\mathbb{R}^{2}$, Studia Math. 51 (1974), 169-182. MR 52:6299

Department of Mathematics, Florida State University, Tallahassee, Florida 323064510

E-mail address: oberlin@math.fsu.edu 